\title{
A Case of Pediatric Paratesticular Rhabdomyosarcoma Misdiagnosed as Epididymitis
}

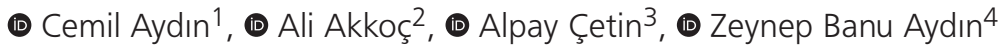 \\ 1 Hitit University Çorum Erol Olçok Training and Research Hospital, Clinic of Urology, Çorum, Turkey \\ 2 Alanya Alaaddin Keykubat University Faculty of Medicine, Department of Urology, Alanya, Turkey \\ ${ }^{3}$ Diyarbakır Gazi Yaşargil Training and Research Hospital, Clinic of Pathology, Diyarbakır, Turkey \\ 4 Hitit University Çorum Erol Olçok Training and Research Hospital, Clinic of Radiology, Çorum, Turkey
}

\begin{abstract}
Paratesticular embryonal rhabdomyosarcoma (RMS) is a rare tumor arising from the mesenchymal tissues of testis and paratesticular tissue. RMS which usually presents as a painless mass in the scrotum is frequently seen in children, teens and young adults. Accurate diagnosis and early treatment are essential as it is an aggressive tumor with high metastatic potential and poor prognosis. Orchiectomy is the treatment of choice. Chemo-radiotherapy is recommended in case of recurrence and metastasis. We aimed to present an anusual case of a 9-year-old child with paratesticular RMS, who presented with a painful scrotal swelling which was misdiagnosed as epididymitis.

Keywords: Rhabdomyosarcoma, embryonal, epididymitis
\end{abstract}

\section{Introduction}

Paratesticular embryonal rhabdomyosarcoma (RMS) is a rare tumor originated from the mesenchymal tissues of testis and paratesticular tissue frequently seen in children, teens and young adults (1). Among the malignant tumors, the most common histotype is liposarcoma (46.4\%), followed by leiomyosarcoma (LMS) (20\%), malignant fibrous histiocytoma (MFH) (13\%), and embryonal RMS (9\%) (2). Paratesticular embryonal RMS constitutes $7 \%$ of all RMS (3). Paratesticular embryonal RMS usually presents as a painless mass in the scrotum (4). However, we herein presented an unusual 9-year-old child with a painful scrotal swelling which was misdiagnosed as epididymitis and caused delay in diagnosis of paratesticular RMS.

\section{Case Presentation}

A 9-year old boy presented with a painful left testicular mass in the left scrotum. He was admitted to the emergency department. The medical history of the patient was unremarkable. Physical examination revealed a moderate tenderness and erythema of the left hemi-scrotum. Scrotal ultrasound showed a large iso-hypoechoic testicular mass measuring $4 \times 3.5 \times 3.5 \mathrm{~cm}$ in the left scrotum with marked increased vascularity and it was suspicious for severe epididymitis and the boy was discharged with antibiotics and instructions to follow up in case symptoms persisted. Ten days later, he was re-admitted to the urology outpatient clinic with persistent pain and swelling. Repeated ultrasound findings showed a testicular tumor measuring $4 \times$ $4 \times 4 \mathrm{~cm}$ in the left scrotum. He was hospitalized for further evaluation and an exploratory operation. Abdominopelvic computed tomography (CT) and chest radiography revealed no metastases. Complete blood count, beta-human chorionic gonadotropin $(0.1 \mathrm{IU} / \mathrm{L})$, serum alpha-fetoprotein $(0.98 \mathrm{ng} / \mathrm{mL})$, and lactate dehydrogenase (110.0 IU/L) were within normal limits. Informed consent form was obtained from the patient's parents. The following day, the patient underwent left high radical orchiectomy. Macroscopic examination showed that the testis was enlarged and there was a yellow myxoid solid tumor $(4 \times 4.2 \times 3.5 \mathrm{~cm})$ (Figure 1). Normal testicular tissue was expelled peripherally. The tunica vaginalis had been invaded by the tumor in a small area but the tunica albuginea and testis had not been invaded by the tumor, and the surgical margin was also negative. Histopathological evaluation of the surgical specimen demonstrated a tumoral proliferation with a mixture of haphazardly arranged rhabdomyoblasts and undifferentiated primitive cells which were small and round with minimal cytoplasm and dark nuclei. Spindle cells, clear cell change and tadpole cells were also seen. The tumor showed a high mitotic activity (Figure 2). Immunohistochemically, the tumor was

Cite this article as: Aydın C, Akkoç A, Çetin A, Aydın ZB. A Case of Pediatric Paratesticular Rhabdomyosarcoma Misdiagnosed as Epididymitis. Bull Urooncol 2020;19(2):92-95 
strongly positive for desmin, vimentin, myoglobin and negative for calretinin, CK19, EMA, AFP, HCG, CD30, CD117, and CD99 (Figure 3).

Because there was no evidence of metastasis and the patient was $<10$ years old, retroperitoneal lymph node dissection (RPLND) was not performed in our case. First, the patient was classified in the group I of the Intergroup RMS study and then 3 cycles of adjuvant chemotherapy (VAC regimen: vincristine 1.5 $\mathrm{mg} / \mathrm{m}^{2}$ on day 8 intravenous (IV); dactinomycin $1.5 \mathrm{mg} / \mathrm{m}^{2}$ on day $8 \mathrm{IV}$; and cyclophosphamide $150 \mathrm{mg} / \mathrm{m}^{2}$ intramuscular on day $1 \sim 7$ ) were performed. At 1 year of follow-up, our patient was assessed and demonstrated good clinical improvement and was determined to be cured.

\section{Discussion}

Paratesticular RMS accounts for $6 \%$ of all non-germinal intrascrotal tumors (2). Primary paratesticular RMS are seen rarely (1). Paratesticular sarcomas are mostly RMS and seen in children (3). There are two frequency peaks for the development of RMS at the age of 4 and 18 years and $70 \%$ of the patients develop under the age of 10 years (3). There is no difference between races in terms of frequency (5). Most of the patients typically present with painless unilateral scrotal swelling like a solid testicular tumor. However, our case presented with a painful unilateral scrotal swelling suggesting epididymitis. When evaluating a patient with acute testicular pain and swelling, the diagnosis should be testicular torsion until proven otherwise. Whereas, testicular torsion usually

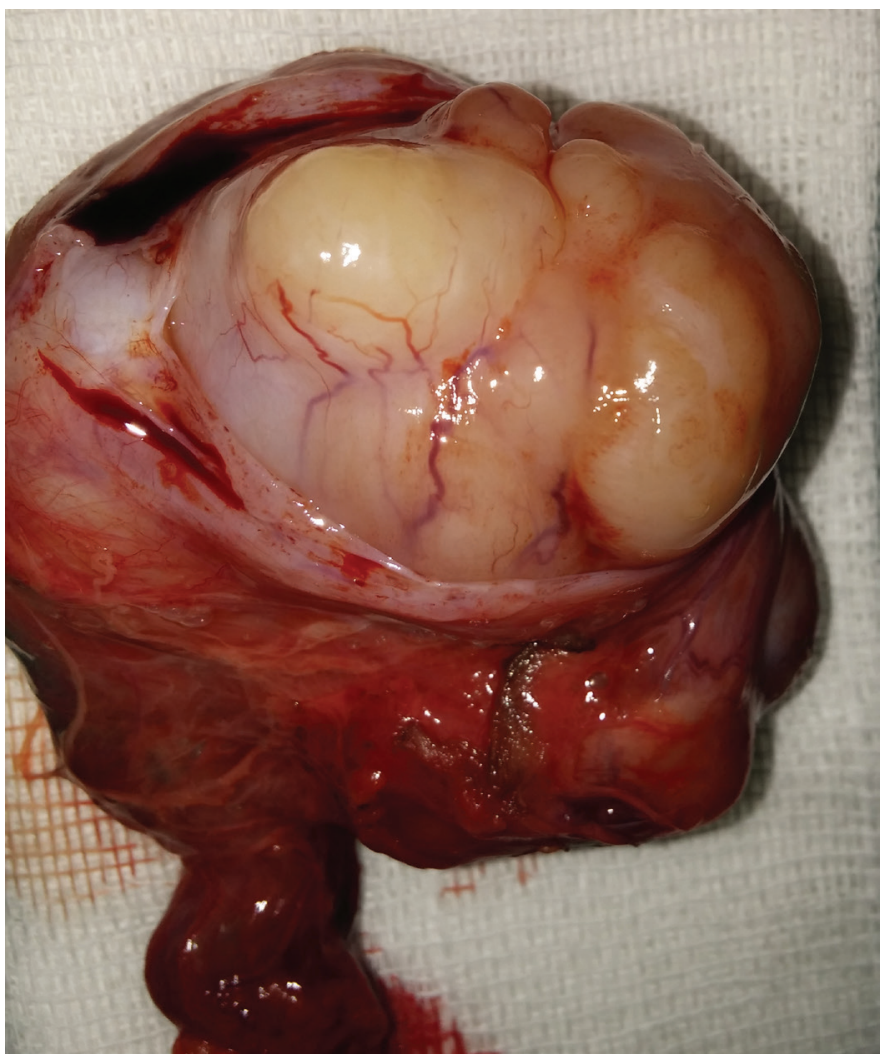

Figure 1. Macroscopic appearance of paratesticular rhabdomyomsarcoma presents as rapid-onset pain, epididymitis and orchitis have a gradual onset of pain. Patients with a testicular tumor usually present with a painless mass found either by the patient or by the physician on routine examination. After completion of a detailed history, the physical examination usually reveals a small nontender palpable lesion on the posterior aspect of the affected testicle. Once a lesion is identified, the scrotum should be transilluminated. A solid mass will not allow light to pass through or give a red glow. A secondary examination of the chest should be performed to evaluate for gynecomastia and abdomen should also be evaluated. Follow-up testing includes diagnostic imaging and laboratory testing. Scrotal ultrasonography will help define suspected lesions revealing a hypoechoic signal. Magnetic resonance imaging can also be employed and will demonstrate a mass that is relatively isointense on T1 imaging and enhancement with IV gadolinium on T2 imaging. Obtaining tumor markers, $\alpha$ fetoprotein, human chorionic gonadotropin, and lactate dehydrogenase also is useful for monitoring progression but cannot be used to screen or to make an initial diagnosis. In all patients with suspected epididymitis, scrotal color Doppler ultrasonography is the preferred test and it should be performed routinely to exclude a scrotal mass. This imaging modality shows a mass with heterogeneous echogenicity and inguinoscrotal extension in $80 \%$ of patients. Sonographic characteristics of paratesticular RMS are nonspecific. An associated hydrocele might be present but is more commonly seen with epididymitis like in our patient. Metastases typically develop by hematogenous or

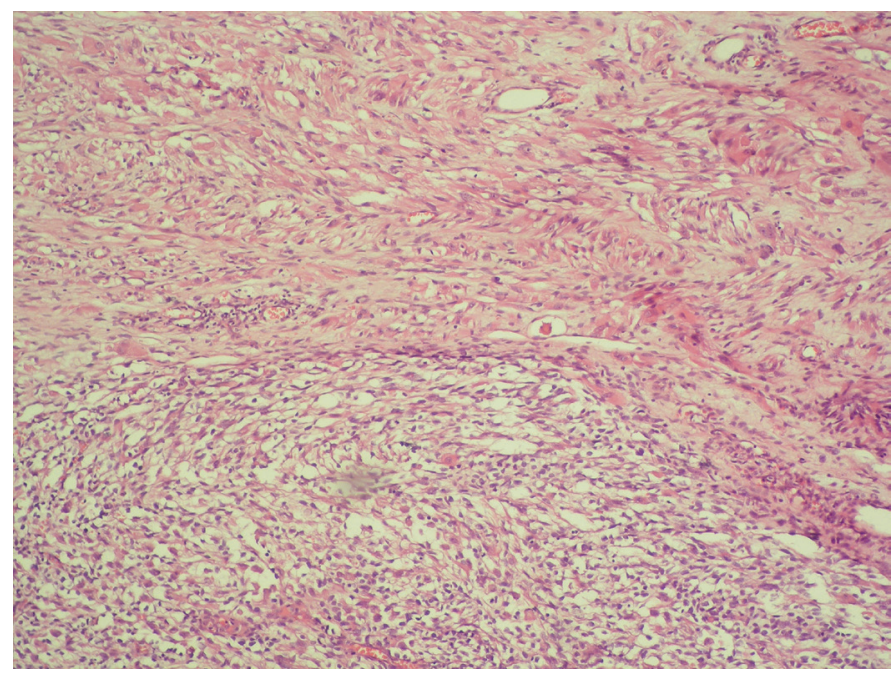

Figure 2. Undifferentiated primitive cells, spindle cells, clear cell change, and rhabdomyoblasts (H\&E x100)
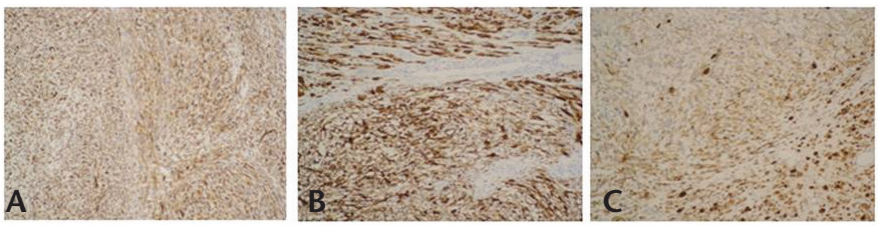

Figure 3. (A) Desmin positivity (x 400),

(B) Vimentin positivity ( $x 400)$,

(C) Myoglobin positivity (x 400) 
lymphatic routes and direct invasion of the testicular tunica (6). Metastasis of RMS is common to the lung, bone marrow, brain, liver, omentum and lymph nodes $(3,6)$. CT scan of chest, abdomen and pelvis is done to rule out possibility of metastasis and most clinicians prefer the use of thoraco-abdomino-pelvic CT compared to ultrasound for regional lymph nodes. In the international classification of RMS, there are 5 recognized variants: Embryonal, alveolar, botryoid embryonal, spindle cell embryonal, and anaplastic (7). The most common variant is embryonal variant, which is most associated with tumors of the genitourinary tract and the head and neck. In embryonal RMS, small cells with hyperchromatic nuclei, minimal cytoplasm as well as cells with rims of eosinophilic cytoplasm and spindle cells with cytoplasmic tails and variable cross striations in myxoid or collagenous stroma is seen. Immunohistochemistry is helpful to exclude other intratesticular spindle cell sarcomas like fibrosarcoma and LMS. Intratesticular RMS have better prognosis than paratesticular RMS (8). Tumor markers such as alpha feto protein, beta-human chorionic gonadotropin and carcinoembryonic antigen are within normal range in RMS. The tumor markers were also within normal range inour patient.

Multidisciplinary treatment approaches including surgery, chemotherapy and radiation have greatly improved the prognosis of paratesticular RMS $(8,9)$. The initial treatment for a RMS is inguinal orchiectomy. Orchiectomy followed by chemotherapy is the best treatment (9). A retroperitoneal lymphadenectomy should not be performed without first obtaining imaging including a CT scan. Because of RMS is chemosensitive, chemotherapy should be routinely performed. The chemotherapy agents used are vincristine, actinomycin D and cyclophosphamide. Radiotherapy is recommended to treat residual microscopic tumor, local recurrence or metastasis (9).

Paratesticular RMS is a rare and potentially aggressive tumor seen in children and young adults. A multimodality approach in diagnosis as well as in treatment is needed. Localized form has good prognosis whereas metastatic disease has poor prognosis. Paratesticular RMS has a better prognosis than RMS originating in other locations because of its favorable histology and possible early detection (8). There is a relationship between age at diagnosis and likelihood of regional lymph node involvement in boys with non-metastatic paratesticular RMS and nodal relaps rate is lower under 10 years of age (10). RMS study group recommends performing a modified ipsilateral retroperitoneal lymph-node dissection over 10 years of age who have no clinical evidence of regional node involvement (10). Although not routinley performed by many centers there have been important debates about the importance of systemic lymphadenectomy because lymph node involvement may accompany $19-38 \%$ of tumors . Abhijith et al. (11) reported that patients over 10 years, with or without radiographic evidence of retroperitoneal disease, should undergo a staging RPLND and receive radiation in addition to chemotherapy if lymph node involvement was positive. The current trend is to avoid dissection if radiological examinations show up no lymph node involvement $>1 \mathrm{~cm}$ (12). Wiener et al. (13) suggested that adolescents should have ipsilateral RPLND dissection as part of their routine staging, and those with positive lymph nodes require intensified chemotherapy as well as nodal irradiation.
In recent years, increased use of minimally invasive surgery has changed to RPLND. Tomaszewski et al. (14) concluded that laparoscopic RPLND for high-risk pediatric patients with paratesticular RMS was a safe diagnostic and therapeutic procedure with the benefit of rapid convalescence, enabling early commencement of adjuvant chemotherapy. Cost et al. (15) reported on two cases of adolescents who were treated using robot-assisted laparoscopic RPLND (R-RPLND), one with paratesticular RMS and one with testicular germ cell tumor with good outcomes and low morbidity. Robotic assistance provides advanced three-dimensional visualization and more precise surgical instrumentation. We hope this procedure will achieve a complete resection and potentially better nerve preservation. Early identification of paratesticular RMS is critical. Radical orchiectomy followed by chemotherapy is the recommended treatment. Radiotherapy is recommended for the control of local recurrence and metastasis. Strict follow up for long term is the rule in all patients.

\section{Acknowledgements}

Publication: The results of the study were not published in full or in part in form of abstracts.

Contribution: There is not any other contributors who may not be listed as authors.

Conflict of Interest: No conflict of interest was declared by the authors.

Financial Disclosure: The authors declared that this study received no financial support.

\section{Ethics}

Informed Consent: Informed consent form was obtained from the patient's parents.

Peer-review: Externally peer-reviewed.

\section{Authorship Contributions}

Concept: A.A., Design: C.A., Data Collection or Processing: A.Ç., Analysis or Interpretation: C.A., Z.B.A., Literature Search: A.A., A.Ç., Writing: C.A.

\section{References}

1. Joan C. Delto, George F. Wayne, Sofia Garces, et al. An adult case of paratesticular spindle cell rhabdomyosarcoma. Urology Case Reports 2014;2:126-128.

2. Rodríguez D, Barrisford GW, Sanchez A, et al. Primary spermatic cord tumors: disease characteristics, prognostic factors, and treatment outcomes. Urol Oncol. 2014;32:19-25.

3. Stewart LH, Lioe TF, Johnston SR. Thirty-year review of intrascrotal rhabdomyosarcoma. Br J Urol 1991;68:418-420.

4. Radouane B, El Fenni J, Chaouir S, et al. Rhabdomyosarcome paratesticulaire: à propos d'un cas. Journal de Radiology 2004;85:779-781.

5. Masson B], Kier R. Sonographic and MR imaging appearances of paratesticular rhabdomyosarcoma. AJR Am J Roentgenol 1998; 171:523-524.

6. Akbar SA, Sayyed TA, Jafri SZ, et al. Multimodality imaging of paratesticular neoplasms and their rare mimics. Radiographics 2003;23:1461-1476. 
7. Qualman S, Lynch J, Bridge J, et al. Prevalence and clinical impact of anaplasia in childhood rhabdomyosarcoma: a report from the Soft Tissue Sarcoma Committee of the Children's Oncology Group. Cancer. 2008;113:3242-3247.

8. Stewart LH, Lioe TF, Johnston SR. Thirty-year review of intrascrotal rhabdomyosarcoma. Br J Urol 1991;68:418-420.

9. Liu ZW, Zhang XQ, Hou GL, et al. Primary adult intratesticular rhabdomyosarcoma: Results of the treatment of six cases. Int J Urol 2011;18:171-174.

10. R. Beverly Raney, Harold M. Maurer, James R, et al. The Intergroup Rhabdomyosarcoma Study Group (IRSG): major lessons from the IRS-I through IRS-IV studies as background for the current IRS-V treatment protocols. Sarcoma 2001;5:9-15.

11. Abhijith SM, Nerli RB, Weiss D, Srinivasan A. Lararoscopic retroperitoneal lymph node dissection for paratesticular rhabdomyosarcoma in older children/adolescents. Indian J Surg Oncol 2013;4:341-344.
12. Ahsaini M, Kassogué A, Tazi MF, et al. Early local recurrence of a pure embryonal rhabdomyosarcoma of the testis successfully managed with local resection associated with lymphadenectomy and adjuvant chemotherapy. J Afr Cancer 2014;6:115-118.

13. Wiener ES, Anderson JR, Ojimba Jl, et al. Controversies in the management of paratesticular rhabdomyosarcoma: is staging retroperitoneal lymph node dissection necessary for adolescents with resected paratesticular rhabdomyosarcoma? Pediatr Surg $2001 ; 146-152$.

14. Tomaszewski JJ, Sweeney DD, Kavoussi LR, et al. Laparoscopic retroperitoneal lymph node dissection for high-risk pediatric patients with paratesticular rhabdomyosarcoma. J Endourol 2010;24:31-34.

15. Cost NG, Dajusta DG, Granberg CF, et al. Robot-assisted laparoscopic retroperitoneal lymph node dissection in an adolescent population. J Endourol 2012;26:635-640. 